the miR-196 miRNAs, whose genes are evolutionarily conserved at the same relative positions in the HoxA, HoxB and HoxC clusters. In particular, they described the presence of target UTR sequences in the closely related Hoxb8, Hoxd8, Hoxc8 and Hoxa7 transcripts and showed, in the case of Hoxb8, that miR-196 could direct cleavage of this transcript. While their results imply that miRNAs may have a role in the colinear distribution of Hox transcripts, neither the presence of miR-196 nor its effect on the Hoxb8 mRNA was determined in the context of the developing embryo.

\section{Profiting from colinearity}

Using transgenic sensors, Mansfield et al. now show that both miR-196a and miR-10a are distributed in Hox-like patterns in the embryo (Fig. 1). This illustrates the advantage of having these miRNA genes embedded in the Hox complexes; only in this location can they be assured of having a similar colinear activation as their neighboring Hox genes. Notably, this phenomenon was also shown in previous studies in which promoters introduced into Hox clusters tend to adopt colinear regulation. In the case of miR-10a, the sensor technique showed that the anterior limits of its expression and that of its genomic neighbor Hoxb4 are the same. In contrast, maximal expression of miR-196a, as judged by weaker lacZ staining, was observed posteriorly, precisely where Hoxb8 mRNA is less abundant, suggesting that miRNAs could participate in the fine regulation of at least some Hox expression patterns. Because miR-196a target UTR sequences are found in all group $8 \mathrm{Hox}$ transcripts, miR-196a may negatively regulate either the steady-state levels or the translational efficiency of all group 8 Hox transcripts in the caudal part of the embryo. This possibility is supported by in vitro work showing that miR-196a can direct processing of the Hoxb8 transcript ${ }^{3,9}$.

Although these observations might bring new players into the complex game of colinearity, it is not yet obvious how this element will contribute to the puzzle's solution. First, the presence of miRNAs and related UTR target sequences seems to be restricted to only a few Hox genes. Therefore, it seems unlikely that this mechanism is a global determinant of Hox expression patterns. Second, transcripts of many Hox/lacZ transgenes are localized quite faithfully even without their corresponding Hox 3' UTRs. Third, the functional relevance of miR-196 has yet to be documented genetically. In worms and flies, loss-of-function mutations have clearly shown that specific miRNAs have a role in development ${ }^{7,10}$. Engineering similar mutations in mice (e.g., by microdeletion) will be a difficult task given the presence of related miRNAs on three of the four Hox clusters. An alternative approach would be to upregulate Hoxb8 in the posterior portion of the embryo (e.g., by expressing a transgene carrying a deletion of the target UTR sequence) to assess the effect, if any, of high steady-state levels of Hoxb8 posteriorly. Our current knowledge of the system suggests that
Hox gain of function posteriorly should not markedly alter morphologies, but such experiments may uncover some surprises.

So far, the only target of the miR-196 or miR-10 RNAs that has been demonstrated in vivo is the miR-196a-mediated degradation of Hoxb8. This makes it tempting to assume that the only targets of these miRNAs are the Hox transcripts. As miRNAs generally have multiple targets, however, the miRNAs in the Hox complexes could synergistically influence $H o x$ function by targeting other mRNAs.

miRNAs are now entering the field of vertebrate development. It is particularly notable that they do so in a genetic system in which the control of timing is $\mathrm{crucial}^{8}$, given that the founding members of this class of molecules are involved in tightly time-controlled processes in Caenorhabditis elegans ${ }^{1}$. Further analyses will determine whether these small RNA molecules have a related function in the precise temporal regulation of Hox gene transcripts.

1. Pasquinelli, A.E. \& Ruvkun, G. Ann. Rev. Cell Dev. Biol. 18, 495-513 (2002).

2. Griffiths-Jones, S. Nucleic Acids Res. 32 , D109-D111 (2004).

3. Mansfield, J.H. et al. Nat. Genet. 36, 1079-1083 (2004).

4. Lagos-Quintana, M. et al. RNA 9, 175-179 (2003).

5. Krichevsky, A.M. et al. RNA 9, 1274-1281 (2003).

6. Chen, X. Science 303, 2022-2025 (2004).

7. Brennecke, J. et al. Cell 113, 25-36 (2003).

8. Kmita, M. \& Duboule, D. Science 301, 331-333 (2003).

9. Yekta, S., Shih, I.H. \& Bartel, D.P. Science 304, 594-596 (2004).

10. Reinhart, B.J. et al. Nature 403, 901-906 (2000).

\title{
How to make an ape brain
}

\author{
Ajit Varki
}

\begin{abstract}
Many genes and genetic mechanisms contributed to the evolution of humans from a common primate ancestor. Emergence of the ape brain was apparently facilitated by a retrotransposed gene duplicate that acquired brainspecific expression and functions affecting the neurotransmitter glutamate.
\end{abstract}

From an anthropocentric perspective, the origin of humans is one of our greatest unsolved mysteries. There are many approaches to explaining the human phenomenon, each with its own problems and prospects. One approach is to compare our

Ajit Varki is at the Glycobiology Research and Training Center, Departments of Medicine and Cellular \& Molecular Medicine, University of California San Diego, La Jolla, California 92093-0687, USA.

e-mail:alvarki@ucsd.edu genomes and genes with those of closely related species, hoping to identify changes that might explain unusual features of the human condition ${ }^{1}$. Hence, there was much excitement about the recent release of the draft sequence of the chimpanzee genome and anticipation about what one might find in comparisons with the human genome ${ }^{2}$. Detailed comparisons of single homologous chimpanzee and human chromosomes ${ }^{3}$, and preliminary reports regarding the whole genome $e^{4}$, indicate that the situation will be far from simple and that we need to search for many needles in a very large haystack of differences. Thus, an important parallel approach is to study select candidate genes. It is also logical to focus on genes that seem to be important in anatomic and physiological systems that show the most unusual human features, including the skin, musculoskeletal system, female reproductive system, immune response and brain. Only in the last decade or so have a number of such candidate genetic differences emerged. Examples run the gamut from outright gene inactivations or deletions to 
Figure 1 An anthropocentric view of primate gene evolution. From the limited data available, many genes underwent substantial changes in the primate lineage leading to humans. As the examples presented here indicate, these changes range from complete gene deletions to subtle changes in amino acid sequences altering function to changes in expression levels of the encoded protein or its enzymatic product. There are many more examples in the literature of human-specific changes in gene expression, mostly defined at the mRNA level. GLUD2 is an unusual example of a gene that emerged as an intronless duplicate of GLUD1 by retrotransposition of its $\mathrm{mRNA}$, achieved specialized expression in the brain and then acquired multiple amino acid changes to facilitate its functioning in the brain. Along with changes in other molecules, such as APSM, MCPH1 and multiple components of the mitochondrial electron transport chain, the emergence of GLUD2 probably facilitated encephalization and specialization of the ape brain, forming a vital basis for the later emergence of the human brain. General facts about most gene names can be found at http://www.gene.ucl.ac.uk/nomenclature/ and literature references at PubMed (http://www. ncbi.nlm.nih.gov/entrez/query.fcgi).

changes in amino acids essential to function to alterations in expression patterns of otherwise similar genes (Fig. 1).

Although much attention is focused on the genetic differences between humans and our closest evolutionary relatives (chimpanzee, bonobo, gorilla and orangutan - the great apes; Fig. 1), one must not forget that this lineage arose from deeper phylogenetic roots that were also quite specialized relative to other mammals - the order of primates in general and the Old World primates (OWPs) in particular. As Goodman, Grossman and colleagues emphasize ${ }^{5}$, it may be just as important to define genetic changes specific to the emergence of the OWPs, without which the final specializations that gave rise to humans would not have had any place to take root. In this regard, it is of note that the vaunted 'big brain' of humans arose on a background of substantial encephalization and specialization that was already ongoing in OWPs. In this context, recent data indicate that several genes known to be important in the structure and function of the human brain have been undergoing positive darwinian selection during and since the emergence of the OWPs, such as those encoding several mitochondrial electron transport chain proteins ${ }^{5}$ and determinants of brain size, such as $A S P M^{6-8}$ and microcephalin $(M C P H 1$; ref. 9). On page 1061 of this issue, Burki and Kaessmann ${ }^{10}$ introduce another player which specifically affects the brain metabolism of

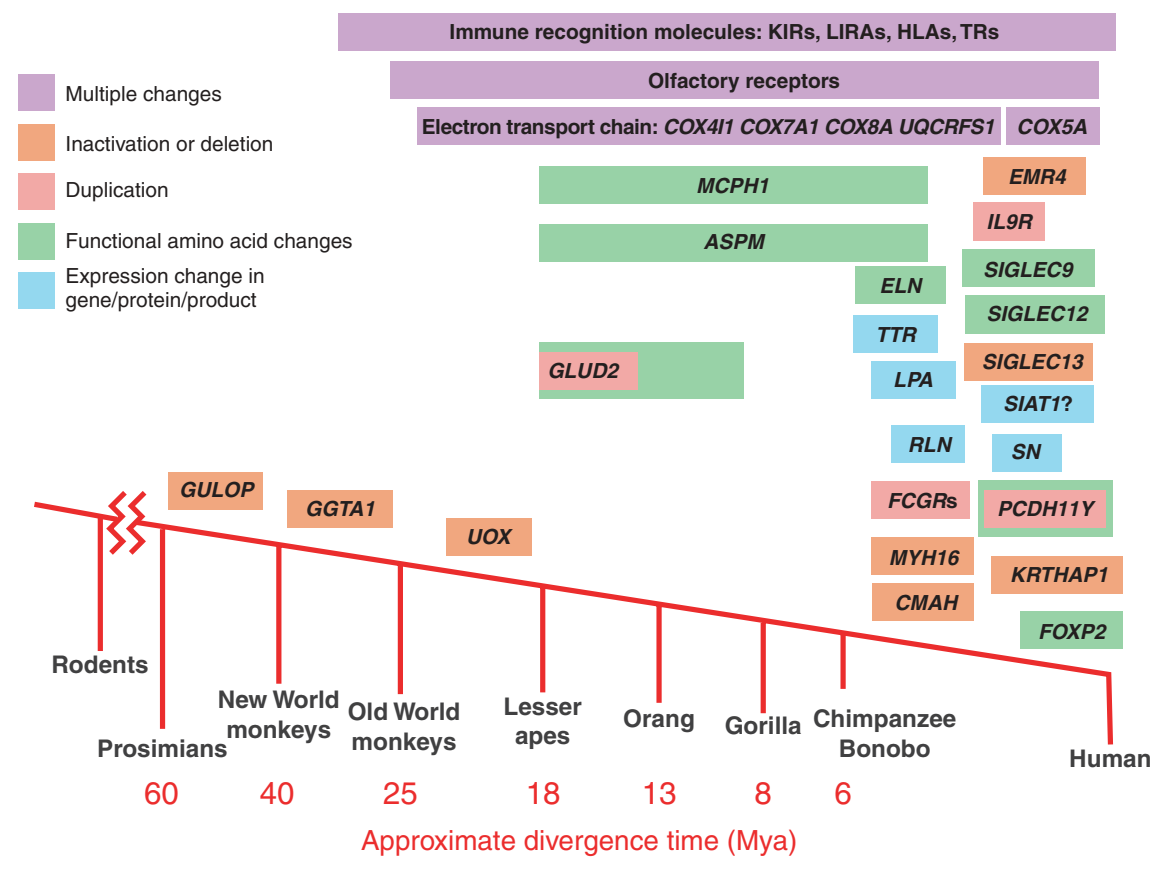

the key neurotransmitter glutamate. The story is made all the more fascinating by the fact that it involves the 'birth' of a new gene in the ape lineage.

\section{Two GLUDs are better than one}

Glutamate dehydrogenase is an enzyme that catalyzes the reversible oxidative deamination of glutamate to $\alpha$-ketoglutarate using NAD or NADP as cofactors. Before 1994, only one mRNA for this enzyme activity was known, encoded by an evolutionarily conserved, introncontaining, widely expressed 'housekeeping' gene called GLUD1. Shashidharan et al. ${ }^{11}$ then discovered a new mRNA that arose from an intronless duplicate of this gene, called GLUD2, and noted it to be selectively expressed in the retina, testis and brain. Although the concept of gene duplication leading to the birth of new genes is well established, such new genes typically arise from small- and large-scale genomic duplication or gene conversion events. In contrast, Burki and Kaessmann ${ }^{10}$ show that GLUD1 underwent duplication by a pathway that more commonly generates processed pseudogenes. Thus, a processed mRNA for GLUD1 became retrotransposed and reinserted itself into the genome as an intronless copy (GLUD2), which acquired a new brain-specific expression pattern and then a series of amino acid sequence changes, leading to the optimization of its functional capabilities in the brain. These functional changes include resistance to the high GTP levels in the brain, a markedly increased activity dependence on the allosteric activators ADP and L-leucine, and the ability to function at relatively low $\mathrm{pH}$. The authors suggest that these features allow instant activation of the enzyme in the brain when there is high frequency firing of neurons.

From the anthropocentric perspective, it is particularly interesting that this event occurred just before the emergence of the ape lineage (Old World monkeys do not have a GLUD2 gene; Fig. 1). Furthermore, Burki and Kaessmann ${ }^{10}$ use various methods of phylogenetic analysis to show that the pattern of amino acid changes in GLUD2 since the duplication event show the signature of positive darwinian selection and that some of them are probably associated with the previously known differences in the biochemical properties of GLUD1 and GLUD2.

\section{Productive insertions}

Such processed pseudogenes seem to be peculiar to mammalian genomes, probably because such genomes have a high content of LINE elements, which provide the reverse transcriptase activity to generate the cDNAs before insertion ${ }^{12,13}$. The occurrence of such processed pseudogene insertions is rather common. For example, comparisons of the chimpanzee and human genomes indicate that $\sim 200$ such insertions have occurred in each of the lineages since their last common ancestor $\sim 6$ million years ago (Mya). Correcting for the current coverage of chim- 
panzee-human alignments and the time of divergence, the estimated rate of formation of processed pseudogenes is 40-60 per Mya (D. Torrents, personal communication, for the Chimpanzee Genome Sequencing Consortium). Most such processed pseudogenes are 'dead on arrival', however, because of copying errors that occur during their creation. Even those that initially contain an intact open reading frame are not typically expressed, because they lack promoters. It is reasonable to speculate that GLUD2 happened by chance to insert adjacent to brain-specific promoter element(s), which then allowed selective pressure to support and enhance its continued expression. Partial deficiency of glutamate dehydrogenase activity has been found in individuals with various neurodegenerative disorders ${ }^{11}$, and excitotoxic neuronal death can be caused by accumulation of excess glutamate ${ }^{14}$. Given that GLUD2 is an ape-specific gene, it is difficult to test its importance in the standard rodent models. As it is located on the X chromosome, however, there is a reasonable likelihood that somewhere in the human population are males who carry GLUD2 mutations. Finding such individuals and studying their phenotypes would be a large step towards understanding the importance of GLUD2 in human brain evolution and function.

Thus, in addition to losing their tails, our ape ancestors seem to have been selected for multiple genetic modifications that favored the emergence of their specialized brains. The current work ${ }^{10}$ provides yet another piece to this part of the complex puzzle of human origins. In the final analysis, genes alone cannot explain the human brain. We must remember that the ape brain in general and the human brain in particular owe many of their sophisticated abilities to a intimate synergy between nature (genes) and nurture (environment). Thus, even if we eventually find all the needles in this haystack, the human mind will ultimately be explained only as "Nature via Nurture"15.
1. Gagneux, P. \& Varki, A. Mol. Phylogenet. Evol. 18, 2-13 (2001).

2. Olson, M.V. \& Varki, A. Nat. Rev. Genet. 4, 20-28 (2003).

3. Watanabe, H. et al. Nature 429, 382-388 (2004).

4. Olson, M.V. \& Varki, A. Science 305, 191-192 (2004).

5. Grossman, L.I., Wildman, D.E., Schmidt, T.R. \& Goodman, M. Trends Genet. (in the press).

6. Zhang, J. Genetics 165, 2063-2070 (2003).

7. Evans, P.D. et al. Hum. Mol. Genet. 13, 489-494 (2004).

8. Kouprina, N. et al. PLoS Biol. 2, E126 (2004).

9. Evans, P.D., Anderson, J.R., Vallender, E.J., Choi, S.S. \& Lahn, B.T. Hum. Mol. Genet. 13, 1139-1145 (2004).

10. Burki, F. \& Kaessmann, H. Nat. Genet. 36, 1061-1063 (2004).

11. Shashidharan, P. et al. J. Biol. Chem. 269, 16971-16976 (1994).

12. Balakirev, E.S. \& Ayala, F.J. Annu. Rev. Genet. 37, 123-151 (2003).

13. Torrents, D., Suyama, M., Zdobnov, E. \& Bork, P. Genome Res. 13, 2559-2567 (2003).

14. Sattler, R. \& Tymianski, M. Mol. Neurobiol. 24, 107-129 (2001).

15. Ridley, M. Nature Via Nurture: Genes, Experience, and What Makes Us Human (HarperCollins, New York, 2003).

\title{
Shifting insulator boundaries
}

\author{
Sangkyun Jeong \& Karl Pfeifer
}

\begin{abstract}
A new study shows that poly(ADP-ribosyl)ation of CTCF regulates its activity as a transcriptional insulator without altering its DNA binding properties. This mechanism provides cells with the flexibility to regulate gene expression without altering fundamental epigenetic programs.
\end{abstract}

Transcriptional insulators are DNA elements that set boundaries on the actions of enhancer and silencer elements and thereby organize the eukaryotic genome into regulatory domains ${ }^{1}$. All vertebrate insulators characterized to date use the versatile CTCF protein. CTCF uses various combinations of its 11 zinc fingers to recognize a variety of unrelated DNA sequences $^{2}$. Once bound to DNA, CTCF can function as a transcriptional insulator, repressor or activator depending on the context of the binding site ${ }^{3}$. CTCF also seems to determine gene expression patterns indirectly by establishing or maintaining specific epigenetic programs in the

\footnotetext{
Sangkyun Jeong and Karl Pfeifer are in the Laboratory of Mammalian Genes and Development, Building 6B-206, National Institute of Child Health and Human Development, National Institutes of Health, Bethesda, Maryland 20892, USA. e-mail:pfeiferk@mail.nih.gov
}

cell $^{3,4}$. On page 1105 of this issue, Yu et al. ${ }^{5}$ identify a post-translational mechanism for regulating CTCF insulator activity that adds to its versatility and its ability to effectively manage epigenetic programs.

Yu et $a l .{ }^{5}$ examine the action of CTCF using the imprinted Igf2-H19 locus as their model system. Figure 1a depicts the organization of the locus, including shared downstream enhancer elements and the H19 imprinting control region (ICR $)^{6}$. Binding of CTCF to its four recognition sites in the ICR prevents enhancer activation of the distal Igf2 promoter but not the proximal H19 promoter, demonstrating the ability of CTCF to act as classical transcriptional insulator. On the paternal chromosome, methylation of the ICR DNA sequences prevents CTCF binding and, therefore, Igf2 is expressed. The demonstration that regulation of CTCF binding could modulate transcriptional insulation was a key finding in both the imprinting and insulator fields.

\section{Let's PARley}

By re-examining the unmethylated (maternal) ICR, Yu et al. ${ }^{5}$ document binding of a poly(ADP-ribosyl)ated protein on this element. They then provide circumstantial but compelling evidence that the poly(ADP-ribosyl)ated protein is CTCF. Poly(ADPribosyl)ation is a protein modification in which homopolymers of up to 200 ADPribose units are attached by the action of poly(ADP-ribose) polymerases (PARPs) ${ }^{7,8}$. As each ADP-ribose has a negative charge, poly(ADP-ribosyl)ation causes a substantial change in the three-dimensional and electrostatic properties of the target protein.

Most notably, Yu et al. ${ }^{5}$ demonstrate that insulator function, but not CTCF binding, is sensitive to the PARP inhibition. That is, blocking PARP activity results in reactivation of the maternal Igf2 gene even while CTCF remains bound to the ICR (Fig. 1b). Thus, this study uncovers a unique mechanism for regulating insulator activity without disrupting CTCF-DNA interactions. A brilliant feature of 